\section{\$32. Bifurcation in Asymmetric Plasma Divided by a Magnetic Filter}

\author{
Naitou, H., Ohi, K., Tauchi, Y., Fukumasa, O. (Yamaguchi \\ Univ. Eng.)
}

A Magnetic filter (MF) is a localized magnetic field usually generated by an array of permanent magnets. The NF installed in the vacuum chamber can separate a plasma into two regions with different parameters. The transport of charged particles across the MIF can be controlled by the properly selected strength of the MF due to the difference of the mass. the charge. and the energy. In this paper, the strength of the MF is chosen to reflect only electrons from both sides of the MF; it has little influence on ion dynamics because of large inertia. Due to thermal insulation of electrons by the MF. a subplasma with low density and low temperature can exist adjacent to a main plasma with high density and high temperature.

The asymmetric plasma divicled by the MF is numerically simulated. The one-dimensional particle-in-cell code VSIM $11 D^{1)}$ is used. Full dynamics of electrons and ions are followed under the electrostatic approximation. Left and right boundaries of the system. $x=0$ and $x=L_{x}=400$, are grounded walls. Particles hitting the walls are absorbed there. The MIF locates at the center of the system $\left(x=x_{\Lambda I F}=200\right)$. The direction of the magnetic field is in the $z$-axis. The spatial profile of the magnetic field strength is given by

$$
B(x)=B_{0} \exp \left[-0.5\left(x-x_{\Lambda F F}\right)^{2} / a_{\Lambda F}^{2}\right],
$$

where $B_{0}=0.5$ and $a_{M F}=12$. The main plasma with $T_{M I e}=4$ and $T_{\Lambda I i}=0.1$ exists in $x_{\Lambda I F} \leq x \leq L_{x}$. whereas the subplasma with $T_{S e}=1$ and $T_{S i}=0.1$ exists in $0 \leq x \leq x_{M I F}$. Here, $T_{N I}$ and $T_{S e}$ are electron temperatures, and $T_{\Lambda I i}$ and $T_{S i}$ are ion temperatures in the respective plasmas. Hydrogen plasma is assumed. Nass ratio is $m_{i} / m_{e}=1836$.

The basic physics observed in this system are reported in references 2 and 3 . Reference 4 has added detailed measurements to clarify the mechanism underlying the physics. We selected $N_{i n}^{-1}$, which is proportional to the plasma production rate in the subplasma. as the parameter to control the asymmetry of the system. Depending on the asymmetry, the system exhibits a static state or a drnamic state. We have observed Hopf bifurcation at the critical point between the static regime and the dynamic regime: the stationary solution changes from the stable fixed point to the stable periodic attractor (limit cycle) as $N_{i n}^{-1}$ reduces. The transition between two bifurcated states is discontinuous at the boundary.

In the dynamic state, the electrostatic potential in the subplasma $\phi_{S}$ shows the self-sustained oscillation.
The minimum and the maximum of $\phi_{S}$ are $\sim 3 T_{S e}$ and $\sim 3 T_{\text {AIe }}\left(T_{S \epsilon}<T_{\text {Me }}\right)$. respectively. The potential in the main plasma $\phi_{M I}$ is almost constant $\left(\sim 3 T_{\text {MIE }}\right)$ with small ripples synchronized with the autonomous oscillation in the subplasma. The modulated ion beam accelerated by the potential gap $\phi_{M I}-\phi_{S}$ around the MF excites the shock wave in the subplasma. The shock wave structure has faster and slower shock fronts. The approaching faster shock front to the grounded wall has the effect to decrease $\phi_{S}$ because the electrons in front of the faster shock front is pushed into the electron-free ion sheath next to the wall. When the slower shock front is absorbed by the wall. $\phi_{S}$ starts increasing due to the difference between the smaller ion flux going into the wall and the larger ion flux coming into the subplasma across the MF.

The reason why the bifurcation is discontinuous at the critical point is explained as follows. We can assume that in the dynamic regime the initial state is the unstable fixed point and the solution of the system moves to the stable attractor. The picture is illustrated as follows. Due to the thermal noise. the potential gap is modulated slightly. The weak shock wave produced by the velocity modulated ion beam reaches the left wall. If the reduction of $\Theta_{S}$ by the approaching shock wave is bigger than that of the former potential modulation. the stronger shock wave is formed. This positive feed back process continues until the maximum potential of the subplasma goes up to $\sim \phi_{M}$. The growth rate of the oscillating part of $\phi_{S}$ is measured in the dynamic regime. The growth rate decreases down to zero at the critical point as $N_{i n}^{-1}$ increases. In the static regime, there is no positive feedback because bulk electrons mitigate the effect of the approaching shock wave. So the threshold exists for the positive feed back to occur. which may be the ratio of the ion beam flux from the main plasma to the plasma density in the subplasma.

In reference 4. the control parameter is fixed during the temporal evolution of the simulation system. It is natural to expect that the system treated here exhibits the hysteresis if the control parameter is changed very slowly. The hysteresis in the asymmetric plasma divided by the MF is studied in reference 5 .

\section{Reference}

1) Koga, K., Naitou, H.. Kawai. Y.. J. Phys. Soc. Japan 68 (1999) 1578.

2) Naitou. H.. Ohi. K.. Fukumasa. (... Rev. Sci. Instrum. $71(2000) 875$.

3) Ohi. K.. Naitou. H.. Tauchi. Y. Fukumasa. O.. Phy. of Plasmas 8 (2001) 23

4) Ohi, K., Naitou, H., Tauchi. Y., Fukumasa. Plasma Phys. Control. Fusion (submitted).

5) Ohi, K., Naitou, H., Tauchi, Y., Fukumasa, O., Itoh, K., J. Plasma Fusion Res. SERIES (submitted). 